\title{
Deforming convex bodies in Minkowski geometry
}

\author{
Vladimir Rovenski* and Paweł Walczak ${ }^{\dagger}$
}

\begin{abstract}
We introduce and study deformation $T_{\boldsymbol{b}, \phi}$ of Minkowski norms in $\mathbb{R}^{n}$, determined by a set $\boldsymbol{b}=\left(\beta_{1}, \ldots, \beta_{p}\right)$ of linearly independent 1 -forms and a smooth positive function $\phi$ of $p$ variables. In particular, the $T_{\boldsymbol{b}, \phi}$-image of a Euclidean norm $\alpha$ is a Minkowski norm, whose indicatrix is a rotation hypersurface with a $p$-dimensional axis passing through the origin. For $p=1$, our deformation generalizes construction of $(\alpha, \beta)$-norm; the last ones form a rich class of "computable" Minkowski norms and play an important role in Finsler geometry. We use compositions of $T_{\boldsymbol{b}, \phi}$-deformations with $\boldsymbol{b}$ 's of length $p$ to define an equivalence relation $\stackrel{p}{\sim}$ on the set of all Minkowski norms in $\mathbb{R}^{n}$. We apply M. Matsumoto result to characterize the cases when the Cartan torsions of a norm and its $T_{\boldsymbol{b}, \phi}$-image either coincide or differ by a C-reducible term.
\end{abstract}

Keywords: Convex body, Minkowski norm, $(\alpha, \boldsymbol{b})$-norm, 1-form, Cartan torsion, C-reducible.

Mathematics Subject Classifications (2010) Primary 51B20; Secondary 58B20

\section{Introduction}

In the paper we introduce and study deformations $T_{\boldsymbol{b}, \phi}$ of Minkowski norms in $\mathbb{R}^{n}$, determined by a sequence $\boldsymbol{b}=\left(\beta_{1}, \ldots, \beta_{p}\right)$ of linearly independent 1 -forms $\beta_{i}$ and a positive function $\phi$ of $p$ variables. In particular, $T_{\boldsymbol{b}, \phi}(\alpha)$ (the images of Euclidean norm $\alpha$ ) are $(\alpha, \boldsymbol{b})$-norms [5], whose indicatrix is a rotation hypersurface with a $p$-dimensional axis passing through the origin. The $(\alpha, \beta)$-norms have also recently been generalized in [3] as homogeneous combinations of several Minkowski norms and one-forms. For $p=1$, our deformations $T_{\beta, \phi}$ generalize construction [6] of $(\alpha, \beta)$-norms, which form a rich class of "computable" Minkowski norms and play an important role in differential geometry. We expect that our $T_{\boldsymbol{b}, \phi}$-deformations will also find many applications in Minkowski Geometry [7] as well as in Finsler Geometry [6]. Our question is about mapping between two pairs $\left(B_{i}, q_{i}\right)(i=1,2), B_{i}$ being a convex body and $q_{i}$ - a point of the interior of $B_{i}$. When $\left(B_{1}, q_{1}\right)$ can be $T_{\boldsymbol{b}, \phi}$-mapped by one-step or a sequence of deformations onto $\left(B_{2}, q_{2}\right)$ ?

More exactly, we pose the following Problem. Consider the space $C p t\left(\mathbb{R}^{n}\right)(n \geq 2)$ of compact pointed subsets of $\mathbb{R}^{n}$ equipped with the Hausdorff distance $d_{H}$. Given two compact convex bodies $B$ (e.g. a ball) and $B^{\prime}$ and points $\mathcal{O} \in B$ and $\mathcal{O}^{\prime} \in B^{\prime}$ in $\mathbb{R}^{n}$, can one approximate (in $C p t\left(\mathbb{R}^{n}\right)$ ) $B^{\prime}$ by the unit ball $\bar{B}=\{\bar{F}=1\}$ of a Minkowski norm $\bar{F}$ having origin $\overline{\mathcal{O}}$ close to $\mathcal{O}^{\prime}$ and being equivalent (in the sense of Definition 4 with either $p=1$ or arbitrary $p$ ) to the norm $F$ of origin $\mathcal{O}$ and $B=\{F=1\}$. That is, given $\epsilon>0$, can one find $\bar{F}$ as above for which $d_{H}\left(B^{\prime}, \bar{B}\right)<\epsilon$ and $\max \left\{F\left(\mathcal{O}^{\prime}-\overline{\mathcal{O}}\right), F\left(\overline{\mathcal{O}}-\mathcal{O}^{\prime}\right)\right\}<\epsilon$. If not, can one do something like that with the Gromov-Hausdorff distance $d_{G H}$ (see [1]) replacing $d_{H}$ ?

In the paper, we define (using compositions of $T_{\boldsymbol{b}, \phi}$-deformations with $\boldsymbol{b}$ 's of length $\leq p$ ) and study an equivalence relation $\stackrel{p}{\sim}$ on the set of all Minkowski norms in $\mathbb{R}^{n}$. We show that any axisymmetric convex body $B$ in $\mathbb{R}^{2}$ can be moved to a unit disc by $T_{\beta, \phi}$-deformation, but we have no example of Minkowski norm in $\mathbb{R}^{2}$ nonequivalent $\stackrel{1}{\sim}$ to Euclidean norm. We prove that any ellipsoid in $\mathbb{R}^{3}$ can be deformed to a sphere in a finite number of steps with $T_{\beta, \phi}$-deformations, but we cannot say the same for a general convex body in $\mathbb{R}^{3}$ with a plane of symmetry. Answers to

\footnotetext{
${ }^{*}$ Department of Mathematics, University of Haifa, e-mail: vrovenski@univ.haifa.ac.il

${ }^{\dagger}$ Katedra Geometrii, Uniwersytet Eódzki, e-mail: pawel.walczak@wmii.uni.lodz.pl
} 
the above questions will require a thorough study of Cartan torsion. In the paper, we characterize the cases when the Cartan torsions of the Minkowski norm $F$ and its image $\bar{F}=T_{\boldsymbol{b}, \phi}(F)$ coincide or their difference is a C-reducible term.

\section{Construction}

Recall that a Minkowski norm on a vector space $\mathbb{R}^{n}(n>1)$ is a function $F: \mathbb{R}^{n} \rightarrow[0, \infty)$ with the properties of regularity, positive 1-homogeneity and strong convexity, see [6]:

$\mathrm{M}_{1}: F \in C^{\infty}\left(\mathbb{R}^{n} \backslash\{0\}\right)$,

$\mathrm{M}_{2}: F(\lambda y)=\lambda F(y)$ for $\lambda>0$ and $y \in \mathbb{R}^{n}$,

$\mathrm{M}_{3}$ : For any $y \in \mathbb{R}^{n} \backslash\{0\}$, the following symmetric bilinear form is positive definite:

$$
g_{y}(u, v)=\frac{1}{2} \frac{\partial^{2}}{\partial s \partial t}\left[F^{2}(y+s u+t v)\right]_{\mid s=t=0}, \quad u, v \in \mathbb{R}^{n} .
$$

By $\mathrm{M}_{2}-\mathrm{M}_{3}, g_{\lambda y}=g_{y}(\lambda>0)$ and $g_{y}(y, y)=F^{2}(y)$. As a result of $\mathrm{M}_{3}$, the indicatrix $S:=\{y \in$ $\left.\mathbb{R}^{n}: F(y)=1\right\}$ is a closed, convex smooth hypersurface that surrounds the origin.

Remark 1. In some interesting cases (when $F$ is not positive definite or smoothly inextendible in some directions, so that these directions must be removed from the domain of $F$ ) Minkowski norms are defined only in conic domains $A \subset \mathbb{R}^{n}$, e.g., 3]. Such cases called pseudo-Minkowski or conic Minkowski norms, are considered below only in examples (e.g., slope or Kropina norms).

The following symmetric trilinear form is called the Cartan torsion for $F$ :

$$
C_{y}(u, v, w)=\frac{1}{4} \frac{\partial^{3}}{\partial r \partial s \partial t}\left[F^{2}(y+r u+s v+t w)\right]_{\mid r=s=t=0}, \quad u, v, w \in \mathbb{R}^{n},
$$

where $y \neq 0$. Note that $C_{y}(u, v, y)=0$ and $C_{\lambda y}=\lambda^{-1} C_{y}$ for $\lambda>0$. A 1-form $I_{y}(u)=$ $\operatorname{Tr}_{g_{y}} C_{y}(u, \cdot, \cdot)$, is called the mean Cartan torsion, its vanishing characterizes Euclidean norm among all Minkowski norms, e.g. 6]. In coordinates, we have

$$
C_{i j k}=\frac{1}{4}\left[F^{2}\right]_{y^{i} y^{j} y^{k}}=\frac{1}{2} \frac{\partial g_{i j}}{\partial y^{k}}, \quad g_{i j}=\frac{1}{2}\left[F^{2}\right]_{y^{i} y^{j}}, \quad C_{k}=C_{i j k} g^{i j},
$$

where $C_{k}$ are components of the mean Cartan torsion. The angular metric tensor of $F$,

$$
K_{y}(u, v)=g_{y}(u, v)-g_{y}(y, u) g_{y}(y, v) / F^{2}(y),
$$

in coordinates has the view $K_{i j}=F \cdot F_{y^{i} y^{j}}=g_{i j}-g_{i p} y^{p} g_{i q} y^{q} / F^{2}$.

A Minkowski norm $F$ is called semi-C-reducible, if its Cartan torsion has the form [6]

$$
C_{i j k}=\frac{p}{n+1}\left(K_{i j} C_{k}+K_{j k} C_{i}+K_{k i} C_{j}\right)+\varepsilon \frac{1-p}{C^{2}} C_{i} C_{j} C_{k},
$$

where $p \in \mathbb{R}$, and

$$
\varepsilon(C)^{2}=g^{i j} C_{i} C_{j} \neq 0 .
$$

$F$ in $\mathbb{R}^{n}(n \geq 3)$ is called $C$-reducible, if its Cartan torsion has the form of (3) with $p=1$,

$$
C_{i j k}=\frac{1}{n+1}\left(K_{i j} C_{k}+K_{j k} C_{i}+K_{k i} C_{j}\right) .
$$

By [4, Proposition 5], any $(\alpha, \beta)$-norm, $F=\alpha \phi(\beta / \alpha)$, with nonzero mean Cartan torsion is semiC-reducible. In dimension greater than two, any C-reducible Minkowski norm has the Randers $(\phi=1+s)$ or the Kropina $(\phi=1 / s)$ type, see [6, Theorem 2.2 (M. Matsumoto)] and Remark 7 . 


\subsection{General case}

Here, we define a deformation of Minkowski norms by using a sequence of $p$ linearly independent 1 -forms in $\mathbb{R}^{n}$ and a positive function of $p$ variables.

Definition 1. Let $F$ be a Minkowski norm on $\mathbb{R}^{n}, \phi: \prod_{i=1}^{p}\left[-\delta_{i}, \delta_{i}\right] \rightarrow(0, \infty)$ a smooth positive function for some $p \leq n$ and $\boldsymbol{b}=\left(\beta_{1}, \ldots, \beta_{p}\right)$ a sequence of linearly independent 1 -forms on $\mathbb{R}^{n}$ of the norm $F\left(\beta_{i}\right)<\delta_{i}$. Then, the $T_{\boldsymbol{b}, \phi^{-}}$deformation of $F$ (or, of convex body defined by $\{F \leq 1\}$ ) is the following mapping:

$$
T_{\boldsymbol{b}, \phi}: F \mapsto F \cdot \phi(s), \quad s=\left(s_{1}, \ldots, s_{p}\right), \quad s_{i}=\beta_{i} / F .
$$

The image $T_{\boldsymbol{b}, \phi}(\alpha)$ of a Euclidean norm $\alpha$ is called $(\alpha, \boldsymbol{b})$-norm, see [5], its indicatrix is a rotation hypersurface in $\mathbb{R}^{n}$ with the $p$-dimensional axis $\operatorname{span}\left\{\beta_{1}^{\sharp}, \ldots, \beta_{p}^{\sharp}\right\}$. For $p=1$ (and $\beta_{1}=\beta$ ), (4) defines the $T_{\beta, \phi}$-transformation, see Section 1.2 and Example 1 below.

Remark 2. Our norm (44) can be viewed as a canonical form of so called $(F, \boldsymbol{b})$-norm, defined by $\bar{F}=\sqrt{L\left(F, \beta_{1}, \ldots, \beta_{p}\right)}$, where $L: \mathbb{R}^{p+1} \rightarrow \mathbb{R}$ is a continuous function with the following properties: a) $L$ is smooth and positive away from $0, \mathrm{~b}) L$ is positively homogeneous of degree 2, i.e., $L(\lambda y)=$ $\lambda^{2} L(y)$ for all $\lambda>0$. Indeed, set $\phi\left(s_{1}, \ldots, s_{p}\right)=\sqrt{L\left(1, s_{1}, \ldots, s_{p}\right)}$, then $\bar{F}=F \phi\left(\beta_{1} / F, \ldots, \beta_{p} / F\right)$. For $p=1$, such combinations were named $\beta$-changes in [2] and $(F, \beta)$-norms in [3, p. 845].

By direct calculation we get

Proposition 1. The composition $T_{\boldsymbol{b}, \phi_{2}} \circ T_{\boldsymbol{b}, \phi_{1}}$ with the same $\boldsymbol{b}$ has the form $T_{\boldsymbol{b}, \phi}$, where

$$
\phi=\phi_{1}(s) \phi_{2}\left(s / \phi_{1}(s)\right) \text {. }
$$

Define real functions of variables $\left(s_{1}, \ldots, s_{p}\right)$ :

$$
\rho=\phi\left(\phi-\sum_{i} s_{i} \dot{\phi}_{i}\right), \quad \rho_{0}^{i j}=\phi \ddot{\phi}_{i j}+\dot{\phi}_{i} \dot{\phi}_{j}, \quad \rho_{1}^{i}=\phi \dot{\phi}_{i}-\sum_{j} s_{j}\left(\phi \ddot{\phi}_{i j}+\dot{\phi}_{i} \dot{\phi}_{j}\right),
$$

where $\dot{\phi}_{i}=\frac{\partial \phi}{\partial s_{i}}, \ddot{\phi}_{i j}=\frac{\partial^{2} \phi}{\partial s_{i} \partial s_{j}}$, etc. Note that the following relations hold:

$$
\dot{\rho}_{i}=\rho_{1}^{i}, \quad \ddot{\rho}_{i j}=\left(\rho_{1}^{i}\right)_{j}^{\prime}=-s_{k}\left(\rho_{0}^{i k}\right)_{j}^{\prime},
$$

and if $s_{i}=0(1 \leq i \leq p)$ then $\rho=1, \rho_{1}^{i}=0$ and $\rho_{0}^{i j}=0$.

Example 1. If $F$ is the Euclidean norm $\alpha(y)=\langle y, y\rangle^{1 / 2}$ then $T_{\beta, \phi}(\alpha)$ is an $(\alpha, \beta)$-norm, see 6 . Some progress was achieved for particular cases of $(\alpha, \beta)$-norms, e.g. Randers norm $\alpha+\beta$, introduced by a physicist G. Randers to study the unified field theory; Kropina norm $\alpha^{2} / \beta$, first introduced by L. Berwald in connection with a Finsler plane with rectilinear extremal, and investigated by V.K. Kropina; slope norm $\frac{\alpha^{2}}{\alpha-\beta}$, introduced by M. Matsumoto to study the time it takes to negotiate any given path on a hillside. These can be viewed as images of $\alpha$ under $T_{\beta, \phi}$-deformations (for $p=1$ ). We define similarly particular $T_{\beta, \phi}$-deformations of Minkowski norms in $\mathbb{R}^{n}$.

(i) The Randers deformation appears for $\bar{F}=F+\beta$ with $F(\beta)<1$, i.e., $\phi(s)=1+s$. We have $\rho=1+s, \rho_{0}=\rho_{1}=1$.

(ii) Generalized Kropina deformations appear for $\bar{F}=F^{l+1} / \beta^{l}(l>0)$, i.e., $\phi(s)=1 / s^{l}(s>0)$. For $l=1$ we get the Kropina deformation. Then $\rho=2 / s^{2}, \rho_{0}=3 / s^{4}, \rho_{1}=-4 / s^{3}$.

(iii) The slope-deformation appears for $\bar{F}=\frac{F^{2}}{F-\beta}$ with $F(\beta)<1$, i.e., $\phi(s)=\frac{1}{1-s}$. We have $\rho=\frac{1-2 s}{(1-s)^{3}}, \rho_{0}=\frac{3}{(1-s)^{4}}$ and $\rho_{1}=\frac{1-4 s}{(1-s)^{4}}$.

(iv) The quadratic deformation appears for $\bar{F}=(F+\beta)^{2} / F$ with $F(\beta)<1$, i.e., $\phi(s)=(1+s)^{2}$. We have $\rho=(1-s)(1+s)^{3}, \rho_{0}=6(1+s)^{2}$ and $\rho_{1}=2(1-2 s)(1+s)^{2}$.

Note that Kropina and slope norms are not Minkowski norms: their $F$ 's are not defined on the whole $\mathbb{R}^{n} \backslash\{0\}$ (have singularities) and their $g_{y}$ 's are not positive definite. Figure 1 shows quadratic deformation of indicatrix of $m$-root norm $F=\left(\left(y^{1}\right)^{m}+\left(y^{2}\right)^{m}\right)^{1 / m}$ in $\mathbb{R}^{2}$ for $m=2, \ldots, 8$ and two cases: a) $0.3 d y^{2}$, and b) $0.3\left(d y^{1}+d y^{2}\right)$ of $\beta$. 


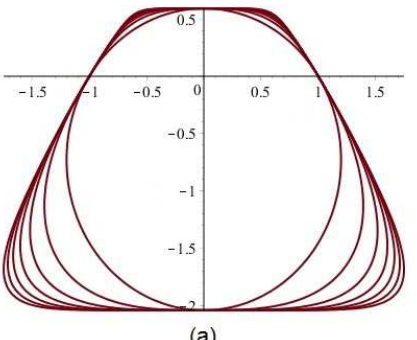

(a)

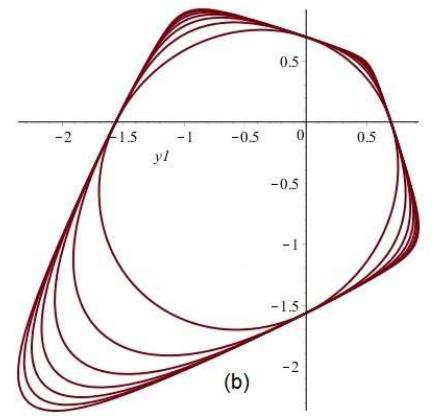

Figure 1: Indicatrix of $m$-root norm in $\mathbb{R}^{2}$ after quadratic deformation.

Assume in the paper that $\rho>0$, i.e.,

$$
\phi-\sum_{i} s_{i} \dot{\phi}_{i}>0
$$

otherwise metric in (7) is not positive for small $s_{i}$. Set $\tilde{p}_{y}=\rho_{1}^{i} p_{y i}$, where $p_{y i}=\beta_{i}^{\sharp y}-s_{i} y / F(y)$.

Theorem 1. Let $\bar{F}=T_{\boldsymbol{b}, \phi}(F)$, then the bilinear forms, see (1), are related as

$$
\begin{aligned}
\bar{g}_{y}(u, v) & =\rho g_{y}(u, v)+\rho_{0}^{i j} \beta_{i}(u) \beta_{j}(v) \\
& +\rho_{1}^{i}\left(\beta_{i}(u) g_{y}(y, v)+\beta_{i}(v) g_{y}(y, u)\right) / F(y)-\rho_{1}^{i} \beta_{i}(y) g_{y}(y, u) g_{y}(y, v) / F^{3}(y) .
\end{aligned}
$$

The Cartan torsions of $\bar{F}$ and $F$, see (2), are related as

$$
\begin{aligned}
& 2 \bar{C}_{y}(u, v, w)=2 \rho C_{y}(u, v, w) \\
& +\left(K_{y}(u, v) g_{y}\left(\tilde{p}_{y}, w\right)+K_{y}(v, w) g_{y}\left(\tilde{p}_{y}, u\right)+K_{y}(w, u) g_{y}\left(\tilde{p}_{y}, v\right)\right) / F(y) \\
& +\sum_{i, j, k}\left(\dot{\phi}_{i} \ddot{\phi}_{j k}+\dot{\phi}_{j} \ddot{\phi}_{i k}+\dot{\phi}_{k} \ddot{\phi}_{i j}+\phi \dddot{\phi}_{i j k}\right) g_{y}\left(p_{y i}, u\right) g_{y}\left(p_{y j}, v\right) g_{y}\left(p_{y k}, w\right) / F(y) .
\end{aligned}
$$

Proof. From (11) and (4) we find

$$
\begin{aligned}
& \bar{g}_{y}(u, v)=\left[\bar{F}^{2} / 2\right]_{F} K_{y}(u, v) / F(y)+\left[\bar{F}^{2} / 2\right]_{F F} g_{y}(y, u) g_{y}(y, v) / F^{2}(y) \\
& +\sum_{i}\left(\left[\bar{F}^{2} / 2\right]_{F \beta_{i}} / F(y)\right)\left(g_{y}(y, u) \beta_{i}(v)+g_{y}(y, v) \beta_{i}(u)\right)+\sum_{i, j}\left[\bar{F}^{2} / 2\right]_{\beta_{i} \beta_{j}} \beta_{i}(u) \beta_{j}(v) .
\end{aligned}
$$

Calculating derivatives of $\frac{1}{2} \bar{F}^{2}=\frac{1}{2} F^{2} \phi^{2}\left(\beta_{1} / F, \ldots, \beta_{p} / F\right)$,

$$
\begin{aligned}
& {\left[\bar{F}^{2} / 2\right]_{F}=F \rho, \quad\left[\bar{F}^{2} / 2\right]_{\beta_{i}}=F \phi \dot{\phi}_{i}, \quad\left[\bar{F}^{2} / 2\right]_{F \beta_{i}}=\rho_{1}^{i}, \quad\left[\bar{F}^{2} / 2\right]_{\beta_{i} \beta_{j}}=\rho_{0}^{i j},} \\
& {\left[\bar{F}^{2} / 2\right]_{F F}=\rho+\left(\sum_{i} s_{i} \dot{\phi}_{i}\right)^{2}+\phi \sum_{i, j} s_{i} s_{j} \ddot{\phi}_{i j}}
\end{aligned}
$$

and comparing (7) and (91), completes the proof of (7). Recall that if $H\left(z^{1}, \ldots, z^{q}\right)$ is a positively homogeneous of degree $r$ function then $H_{z^{k}} z^{k}=r H$. The 0-homogeneity of $\left[\bar{F}^{2} / 2\right]_{\mu \nu}$ in variables $F,\left\{\beta_{k}\right\}$ yields

$$
\left[\bar{F}^{2} / 2\right]_{F \mu \nu} F+\sum_{k}\left[\bar{F}^{2} / 2\right]_{\beta_{k} \mu \nu} \beta_{k}=0
$$

for $\mu, \nu \in\left\{F, \beta_{1}, \ldots, \beta_{p}\right\}$; hence,

$$
\begin{aligned}
& {\left[\bar{F}^{2} / 2\right]_{F \beta_{i} \beta_{j}}=-\sum_{k}\left(\beta_{k} / F\right)\left[\bar{F}^{2} / 2\right]_{\beta_{i} \beta_{j} \beta_{k}},} \\
& {\left[\bar{F}^{2} / 2\right]_{F F \beta_{i}}=\sum_{j, k}\left(\beta_{j} / F\right)\left(\beta_{k} / F\right)\left[\bar{F}^{2} / 2\right]_{\beta_{i} \beta_{j} \beta_{k}},} \\
& {\left[\bar{F}^{2} / 2\right]_{F F F}=-\sum_{i, j, k}\left(\beta_{i} / F\right)\left(\beta_{j} / F\right)\left(\beta_{k} / F\right)\left[\bar{F}^{2} / 2\right]_{\beta_{i} \beta_{j} \beta_{k}} .}
\end{aligned}
$$


Using this, we calculate the Cartan torsion (2) after $T_{\boldsymbol{b}, \phi}$-deformation as

$$
\begin{aligned}
& 2 \bar{C}_{y}(u, v, w)=2 \rho C_{y}(u, v, w) \\
+ & \sum_{i}\left[\bar{F}^{2} / 2\right]_{F \beta_{i}}\left(K_{y}(u, v) g_{y}\left(p_{y i}, w\right)+K_{y}(v, w) g_{y}\left(p_{y i}, u\right)+K_{y}(w, u) g_{y}\left(p_{y i}, v\right)\right) / F(y) \\
+ & \sum_{i, j, k}\left[\bar{F}^{2} / 2\right]_{\beta_{i} \beta_{j} \beta_{k}} g_{y}\left(p_{y i}, u\right) g_{y}\left(p_{y j}, v\right) g_{y}\left(p_{y k}, w\right) .
\end{aligned}
$$

Next, using equalities (10), we obtain

$$
\left[\bar{F}^{2} / 2\right]_{\beta_{i} \beta_{j} \beta_{k}}=\left(\dot{\phi}_{i} \ddot{\phi}_{j k}+\dot{\phi}_{j} \ddot{\phi}_{i k}+\dot{\phi}_{k} \ddot{\phi}_{i j}+\phi \dddot{\phi}_{i j k}\right) / F(y) \text {. }
$$

The above, and comparing (11) and (8) completes the proof of (8).

Remark 3. By (7), $\bar{g}_{y}$ of $T_{\boldsymbol{b}, \phi}$-deformation $\bar{F}$ can be viewed as a perturbed metric $g_{y}$ of $F$. The second line term on the RHS of (8) is the symmetric product of the 2-tensor $K_{y} / F(y)$ and the 1-form $\iota_{\tilde{p}_{y}} g_{y}$ while the third line term is a linear combination of symmetric products of 1-forms $\iota_{p_{y i}} g_{y}(i=1, \ldots, p)$. Therefore, the difference $\bar{C}_{y}-\rho C_{y}$ is semi-C-reducible when $p=1$ (see also [6]), but in general is not when $p>1$.

Proposition 2. Let $\phi$ be a smooth positive function of class $C^{2}$ defined in a neighborhood of the origin $O$ of $\mathbb{R}^{p}$. Then there exists $\delta>0$ such that (6) holds and for any Minkowski norm $F$ and arbitrary 1 -forms $\beta_{1}, \ldots, \beta_{p}$ of $F$-norm less than $\delta$; then (4) determines the Minkowski norm $\bar{F}$.

Proof. The formula (7) shows that the inner products $\bar{g}_{y}$ for $y \in F^{-1}(1)$ depend uniformly on the 1-forms $\beta_{i}(i=1, \ldots, p)$. For $\beta_{1}=\ldots=\beta_{p}=0, \bar{g}_{y}=\rho g_{y}$ is positive definite. Compactness of the $F$-unit sphere implies the statement.

Remark 4. One can get a more accurate than Proposition 2 sufficient conditions for positive definiteness of $\bar{g}_{y}$. By [3, Theorem 4.1], $\bar{g}_{y}>0$ for $\bar{F}=\sqrt{L}=F \phi\left(\frac{\beta_{1}}{F}, \ldots, \frac{\beta_{k}}{F}\right)$, see (44), if (for $\left.L=F^{2} \phi^{2}\right)$
(a) $L_{, F}>0$,
(b) $\operatorname{Hess}(L)>0$ (positive definite matrix).

For $p=1$, we have $L_{, F}>0$ and $\operatorname{Hess}(L)>0$ if and only if, see [3, Eqn. (4.13)],

$$
\phi-s \dot{\phi}>0, \quad \ddot{\phi}>0 .
$$

For $p>1$, we find $L_{, F}=2 F \phi\left(\phi-\sum_{i} s_{i} \dot{\phi}_{i}\right)$, thus, the inequality $L_{, F}>0$ is guaranteed by assumption (6). Calculation of $\operatorname{Hess}(L)$, see condition (b), is similar to the case of $p=1$ in the proof of [3, Proposition 4.10]. Put $L=F^{2} \psi\left(s_{1}, \ldots, s_{p}\right)$, where $s_{i}=b_{i} / F, \psi=\phi^{2}$ and $p>1$. Then $L_{, b_{i}}=F \dot{\psi}_{i}$ and

$$
\begin{aligned}
& L_{, F F}=2 \psi-2 \sum_{i} s_{i} \dot{\psi}_{i}+\sum_{i, j} s_{i} s_{j} \ddot{\psi}_{i j} \\
& L_{, F b_{i}}=\dot{\psi}_{i}-\sum_{j} s_{j} \ddot{\psi}_{i j}, \quad L_{, b_{i} b_{j}}=\ddot{\psi}_{i j} .
\end{aligned}
$$

Applying elementary transformations of matrices to the first row and first column of $\operatorname{Hess}(L)$, we find

$$
\operatorname{Hess}(L) \sim \Psi=\left(\begin{array}{cccc}
2 \psi & \dot{\psi}_{1} & \cdots & \dot{\psi}_{p} \\
\dot{\psi}_{1} & \ddot{\psi}_{11} & \cdots & \ddot{\psi}_{1 p} \\
\cdots & \cdots & \cdots & \cdots \\
\dot{\psi}_{p} & \ddot{\psi}_{1 p} & \cdots & \ddot{\psi}_{p p}
\end{array}\right), \quad \operatorname{det} \operatorname{Hess}(L)=\operatorname{det} \Psi .
$$

In our case, $\operatorname{Hess}(L)>0$ is satisfied when $\operatorname{det} \Psi>0$ and $\psi$ is a convex function. Note that conditions in Proposition 2 do not require $\psi=\phi^{2}$ to be convex. 
We restrict ourselves to regular $T_{\boldsymbol{b}, \phi}$-deformations alone, i.e., $\operatorname{det} \bar{g}_{y} \neq 0(y \neq 0)$. By (77),

$$
\bar{g}_{y}(u, v)=\rho g_{y}(u, v)+\left(\rho_{0}^{i j}+\varepsilon^{-1} \rho_{1}^{i} \rho_{1}^{j}\right) \beta_{i}(u) \beta_{j}(v)-\varepsilon g_{y}(\tilde{Y}, u) g_{y}(\tilde{Y}, v),
$$

where

$$
\tilde{Y}=\varepsilon^{-1} \rho_{1}^{i} \beta_{i}^{\sharp, y}-y / F(y), \quad \varepsilon=s_{j} \rho_{1}^{j} .
$$

The volume forms of metrics $\bar{g}_{y}$ and $g_{y}$ for $y \neq 0$ are

$$
\mathrm{d} \operatorname{vol}_{g_{y}}\left(e_{1}, \ldots, e_{n}\right)=\sqrt{\operatorname{det} g_{y}\left(e_{i}, e_{j}\right)}, \quad \mathrm{d} \operatorname{vol}_{\bar{g}_{y}}\left(e_{1}, \ldots, e_{n}\right)=\sqrt{\operatorname{det} \bar{g}_{y}\left(e_{i}, e_{j}\right)},
$$

where $\left\{e_{1}, \ldots, e_{n}\right\}$ is a basis of $\mathbb{R}^{n}$. Then $\mathrm{d} \operatorname{vol}_{\bar{g}_{y}}=\sigma_{y} \mathrm{~d} \operatorname{vol}_{g_{y}}$ for some function $\sigma_{y}>0$. Define vectors $\tilde{\beta}_{k}=q_{k}^{i} \beta_{i}(k \leq p)$, where $q_{k}=\left(q_{k}^{1}, \ldots, q_{k}^{p}\right) \in \mathbb{R}^{p}$ are unit eigenvectors with eigenvalues $\lambda^{k}$ of the matrix $\left\{\rho_{0}^{i j}+\varepsilon^{-1} \rho_{1}^{i} \rho_{1}^{j}\right\}$. Then (12) takes the form, which can be used to find $\sigma_{y}$ :

$$
\bar{g}_{y}(u, v)=\rho g_{y}(u, v)+\sum_{i} \lambda^{i} \tilde{\beta}_{i}(u) \tilde{\beta}_{i}(v)-\varepsilon g_{y}(\tilde{Y}, u) g_{y}(\tilde{Y}, v) .
$$

Example 2. For $p=2$, using $\tilde{b}_{i j}=g_{y}\left(\tilde{\beta}_{i}, \tilde{\beta}_{j}\right), \tilde{\beta}_{i}=q_{i}^{1} \beta_{1}+q_{i}^{2} \beta_{2}$ and $\varepsilon=\rho_{1}^{1} s_{1}+\rho_{1}^{2} s_{2}$, we get

$$
\begin{aligned}
\sigma_{y} & =\rho^{n-1}\left(\rho^{2}+\rho\left(\lambda^{1} \tilde{b}_{11}+\lambda^{2} \tilde{b}_{22}\right)-\rho \varepsilon g_{y}(\tilde{Y}, \tilde{Y})+\lambda^{1} \lambda^{2}\left(\tilde{b}_{11} \tilde{b}_{22}-\tilde{b}_{12}^{2}\right)\right. \\
& -\varepsilon g_{y}(\tilde{Y}, \tilde{Y})\left(\lambda^{1} \tilde{b}_{11}+\lambda^{2} \tilde{b}_{22}\right)+\lambda^{1} \varepsilon g_{y}\left(\tilde{\beta}_{1}, \tilde{Y}\right)+\lambda^{2} \varepsilon g_{y}\left(\tilde{\beta}_{2}, \tilde{Y}\right)+\lambda^{1} \lambda^{2} \varepsilon / \rho\left[\tilde{b}_{11} g_{y}\left(\tilde{\beta}_{2}, \tilde{Y}\right)^{2}\right. \\
& \left.\left.+\tilde{b}_{22} g_{y}\left(\tilde{\beta}_{1}, \tilde{Y}\right)^{2}+\tilde{b}_{12} g_{y}(\tilde{Y}, \tilde{Y})^{2}-\tilde{b}_{11} \tilde{b}_{22} g_{y}(\tilde{Y}, \tilde{Y})-2 \tilde{b}_{12} g_{y}\left(\tilde{\beta}_{1}, \tilde{Y}\right) g_{y}\left(\tilde{\beta}_{2}, \tilde{Y}\right)\right]\right) .
\end{aligned}
$$

A special case of $T_{\boldsymbol{b}, \phi}$-deformation for $p=2$ is a shifted $T_{\phi, \beta_{1}}$-deformation $\bar{F}=F \phi\left(\beta_{1} / F\right)+\beta_{2}$.

(a) For a shifted Kropina deformation $\bar{F}=F^{2} / \beta_{1}+\beta_{2}$, we get

$$
\begin{array}{ll}
\rho=\left(2+s_{1}\right)\left(1+s_{1}+s_{1} s_{2}\right) / s_{1}^{2}, & \rho_{1}^{1}=-\left(4+3 s_{1}+2 s_{1} s_{2}\right) / s_{1}^{3}, \quad \rho_{1}^{2}=\left(2+s_{1}\right) / s_{1}, \\
\rho_{0}^{11}=\left(3+2 s_{1}+2 s_{1} s_{2}\right) / s_{1}^{4}, & \rho_{0}^{12}=\rho_{0}^{21}=-1 / s_{1}^{2}, \quad \rho_{0}^{22}=1 .
\end{array}
$$

(b) For a shifted slope deformation $\bar{F}=F^{2} /\left(F-\beta_{1}\right)+\beta_{2}$, we have

$$
\begin{array}{ll}
\rho=\frac{\left(1-2 s_{1}\right)\left(1+s_{2}-s_{1} s_{2}\right)}{\left(1-s_{1}\right)^{3}}, \quad \rho_{1}^{1}=\frac{1+2 s_{1}\left(s_{1} s_{2}-s_{2}-2\right)}{\left(1-s_{1}\right)^{4}}, \quad \rho_{1}^{2}=\frac{1-2 s_{1}}{\left(1-s_{1}\right)^{2}}, \\
\rho_{0}^{11}=\left(3-2 s_{1} s_{2}+2 s_{2}\right) /\left(1-s_{1}\right)^{4}, \quad \rho_{0}^{12}=\rho_{0}^{21}=1 /\left(1-s_{1}\right)^{2}, & \rho_{0}^{22}=1 .
\end{array}
$$

Figure 2 shows the image of $\left(y^{1}\right)^{4}+\left(y^{2}\right)^{4}+\left(y^{3}\right)^{4}=1$ in $\mathbb{R}^{3}$ for a) quadratic deformation with $p=1$ and $\left.\beta=0.3 d y^{3}, \mathrm{~b}\right)$ shifted quadratic deformation with $p=2$ and $\beta_{1}=0.3 d y^{2}, \beta_{2}=0.3 d y^{3}$.
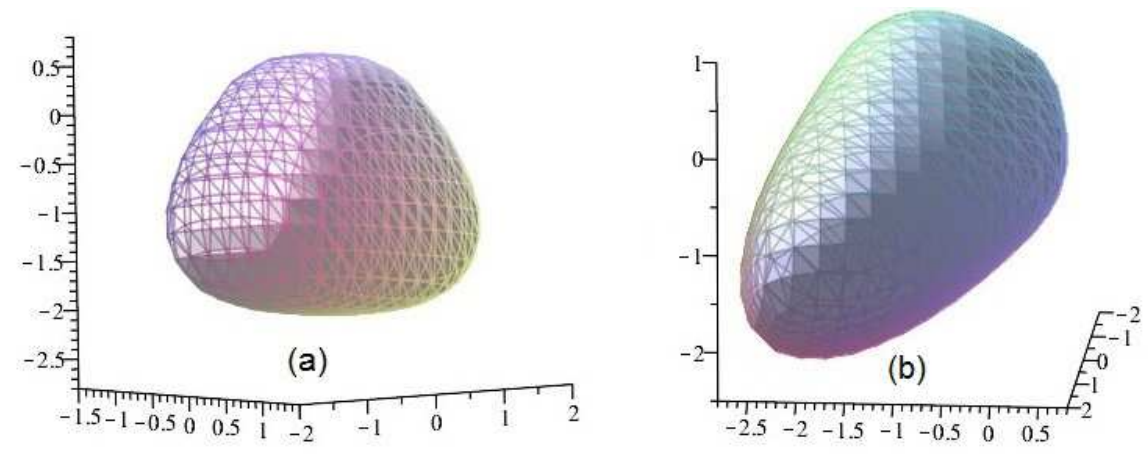

Figure 2: Indicatrix of $m$-root norm in $\mathbb{R}^{3}$ after shifted quadratic deformation.

Definition 2. Let $G$ be a subgroup of $G L(n, \mathbb{R})$. Then a Minkowski norm $F$ on $\mathbb{R}^{n}$ is called $G$-invariant if the following holds for some affine coordinates $\left(y^{1}, \ldots, y^{n}\right)$ of $\mathbb{R}^{n}$ :

$$
F\left(y^{1}, \ldots, y^{n}\right)=F\left(f\left(y^{1}, \ldots, y^{n}\right)\right), \quad y \in \mathbb{R}^{n}, f \in G .
$$


Proposition 3 (see [5]). A Minkowski norm $F$ on $\mathbb{R}^{n}$ is $G$-invariant, where

$$
G=\left\{\left(\begin{array}{cc}
C & 0 \\
0 & \operatorname{id}_{p}
\end{array}\right), \quad C \in O(n-p, \mathbb{R})\right\}
$$

if and only if there exist linear independent 1-forms $\beta_{i}(1 \leq i \leq p)$, for which $F$ is $(\alpha, \boldsymbol{b})$-norm.

Using Proposition 3, we can prove the following.

Corollary 1. Let $F$ and $\bar{F}=T_{\boldsymbol{b}, \phi}(F)$ be Minkowski norms in $\mathbb{R}^{n}$, where $\boldsymbol{b}=\left(\beta_{1}, \ldots, \beta_{p}\right)$ and $\phi$ is a function of $p<n$ variables. Suppose that $F$ is an $(\alpha, \beta)$-norm with 1 -form $\beta=\beta_{p+1}$ transverse to $\operatorname{span}\left\{\beta_{1}, \ldots, \beta_{p}\right\}$. Then $\bar{F}=T_{\boldsymbol{b}, \psi}(\alpha)$, where $\boldsymbol{b}=\left(\beta_{1}, \ldots, \beta_{p+1}\right), \psi$ is a function of $p+1$ variables and $\alpha$ is a Euclidean norm.

\subsection{Case of $p=1$}

Here, we illustrate and clarify some results about our transformations of Minkowski norms for the case $p=1$. By Proposition 3 for $p=1$ and $n=2$, any axisymmetric convex body $B$ in $\mathbb{R}^{2}$ (for example, indicatrix of an $m$-root norm) can be moved to a unit disc by $T_{\beta, \phi}$-deformation.

The next lemma is used to compute the volume forms, it extends the Silvester's determinant identity $\operatorname{det}\left(\mathrm{id}_{n}+C_{1} P_{1}^{t}\right)=1+C_{1}^{t} P_{1}$, where $C_{1}$ and $P_{1}$ are $n$-vectors (columns).

Lemma 1. Given real $c_{1}, c_{2}$, vectors $b^{1}, b^{2}$ in $\mathbb{R}^{n}$, and reversible symmetric $n \times n$ matrix $a_{i j}$, define matrices $A_{i j}=a_{i j}+c_{1} b_{i}^{1} b_{j}^{1}$ and $g_{i j}=a_{i j}+c_{1} b_{i}^{1} b_{j}^{1}+c_{2} b_{i}^{2} b_{j}^{2}$. Then

$$
\begin{aligned}
\operatorname{det}\left[A_{i j}\right] & =\operatorname{det}\left[a_{i j}\right]\left(1+c_{1}\left|b^{1}\right|_{a}^{2}\right), \\
\operatorname{det}\left[g_{i j}\right] & =\operatorname{det}\left[a_{i j}\right] \cdot\left[\left(1+c_{1}\left|b^{1}\right|_{a}^{2}\right)\left(1+c_{2}\left|b^{2}\right|_{a}^{2}\right)-c_{1} c_{2}\left\langle b^{1}, b^{2}\right\rangle_{a}^{2}\right] .
\end{aligned}
$$

Proof. The first claim is straightforward, [6, Lemma 4.1]. If $1+c_{1}\left|b^{1}\right|_{a}^{2} \neq 0$ then the inverse matrix $A^{k l}=a^{k l}-\frac{c_{1}}{1+c_{1}\left|b^{1}\right|_{a}^{2}} b_{k}^{1} b_{l}^{1}$ exists. For any vectors $u, v$ we get

$$
\langle u, v\rangle_{A}=A^{k l} u_{k} v_{l}=\left(a^{k l}-\frac{c_{1} b^{1 k} b^{1 l}}{1+c_{1}\left|b^{1}\right|_{a}^{2}}\right) u_{k} v_{l}=\langle u, v\rangle_{a}-\frac{c_{1}}{1+c_{1}\left|b^{1}\right|_{a}^{2}}\left\langle b^{1}, u\right\rangle_{a}\left\langle b^{1}, v\right\rangle_{a}
$$

Hence,

$$
\left|b^{2}\right|_{A}^{2}=A^{k l} b_{k}^{2} b_{l}^{2}=\left|b^{2}\right|_{a}^{2}-\frac{c_{1}}{1+c_{1}\left|b^{1}\right|_{a}^{2}}\left\langle b^{1}, b^{2}\right\rangle_{a}^{2}
$$

Using the first claim, we get $\operatorname{det}\left[g_{i j}\right]=\operatorname{det}\left[A_{i j}\right]\left(1+c_{2}\left|b^{2}\right|_{A}^{2}\right)$. The above yields the second claim.

We will specify Proposition 2 for $p=1$ (and generalize [6, Lemma 2], that is for $F=\alpha$ ).

Proposition 4 (see Corollary 4.25 in [3]). Let $F=F_{0} /\left(\phi / F_{0}\right)$, choose $y_{0} \in A \backslash 0$ (see Remark 1 and [3, Proposition 4.10]), and put $s_{0}=\beta\left(y_{0}\right) / F_{0}\left(y_{0}\right)$. In the case of dimension $n>2$, the fundamental tensor $g_{y_{0}}$ is positive definite if and only if

$$
\begin{aligned}
& \phi\left(s_{0}\right)-s_{0} \dot{\phi}\left(s_{0}\right)>0, \\
& \phi\left(s_{0}\right)-s_{0} \dot{\phi}\left(s_{0}\right)+\left(\|\beta\|_{g_{y_{0}}^{0}}^{2}-s_{0}^{2}\right) \ddot{\phi}\left(s_{0}\right)>0,
\end{aligned}
$$

and, in the case of $n=2, g_{y_{0}}$ is positive definite if and only if (14b) holds.

The following result is a consequence of Proposition 4. We prove it here just for the convenience of a reader.

Corollary 2. The function $\bar{F}=F \phi(s)$, where $s=\beta / F$, is a Minkowski norm for any Minkowski norm $F$ and a 1-form $\beta$ on $\mathbb{R}^{n}$ with $F(\beta)<b_{0}$, if and only if the function $\phi:\left(-b_{0}, b_{0}\right) \rightarrow \mathbb{R}$ satisfies

$$
\phi(s)>0, \quad \phi(s)-s \dot{\phi}(s)+\left(b^{2}-s^{2}\right) \ddot{\phi}(s)>0,
$$

where $s$ and $b$ are arbitrary real numbers with $|s| \leq b<b_{0}$. 
Proof. Assume that (15) is satisfied. Taking $s \rightarrow b$ in (15), we see that

$$
\phi-s \dot{\phi}>0
$$

for any $s$ with $|s|<b_{0}$. Consider the following families of functions and metrics:

$$
\phi_{t}(s):=1-t+t \phi(s), \quad \bar{F}_{t}=F \phi_{t}(\beta / F), \quad \bar{g}_{y}^{t}: \bar{g}_{i j}^{t}=(1 / 2)\left[\bar{F}_{t}^{2}\right]_{y^{i} y^{j}} .
$$

Note that for any $t \in[0,1]$ and any $s, b$ with $|s| \leq b<b_{0}$,

$$
\begin{aligned}
& \phi_{t}-s \dot{\phi}_{t}=1-t+t(\phi-s \dot{\phi})>0, \\
& \phi_{t}-s \dot{\phi}_{t}+\left(b^{2}-s^{2}\right) \ddot{\phi}_{t}=1-t+t\left\{\phi-s \dot{\phi}+\left(b^{2}-s^{2}\right) \ddot{\phi}\right\}>0 .
\end{aligned}
$$

By Lemma 1, for $\bar{F}_{t}=F \phi_{t}(\beta / F)$ we find a formula (as in the case of $F=\alpha$, see [6])

$$
\operatorname{det} \bar{g}_{y}^{t}=\phi_{t}^{n+1}\left(\phi_{t}-s \dot{\phi}_{t}\right)^{n-2}\left[\phi_{t}-s \dot{\phi}_{t}+\left(b^{2}-s^{2}\right) \ddot{\phi}_{t}\right] \operatorname{det} g_{y} .
$$

Thus, $\operatorname{det} \bar{g}_{y}^{t}>0$ for all $t \in[0,1]$, see also (20) in what follows. Since $\bar{g}_{y}^{0}=g_{y}$ is positive definite, then $\bar{g}_{y}^{t}$ is positive definite for any $t \in[0,1]$. Hence, $\bar{F}_{t}$ is a Minkowski norm for any $t \in[0,1]$. In particular, $\bar{F}=\bar{F}_{1}$ is a Minkowski norm.

Conversely, assume that $\bar{F}=F \phi(\beta / F)$ is a Minkowski norm for any Minkowski norm $F$ and 1-form $\beta$ with $b:=F(\beta)<b_{0}$. Then $\phi(s)>0$ for any $s$ with $|s|<b_{0}$. If $n$ is even, then $\operatorname{det} \bar{g}_{y}>0$ implies that (15) holds for any $s$ with $|s| \leq b$. If $n>1$ is odd, then $\operatorname{det} \bar{g}_{y}>0$ implies that the inequality $\phi(s)-s \dot{\phi}(s) \neq 0$ holds for any $s$ with $|s| \leq b$.

Since $\phi(0)>0$, the above inequality implies that (16) holds for any $s$ with $|s|<b$. Since $b$ with $0 \leq b \leq b_{0}$ is arbitrary, we conclude that (16) holds for any $s$ with $|s|<b_{0}$. Finally, we can see that $\operatorname{det} \bar{g}_{y}>0$ implies that (15) holds for any $s$ and $b$ with $|s| \leq b<b_{0}$.

Definition 3 (see Definition 1 for $p=1)$. Given a smooth positive function $\phi:\left(-b_{0}, b_{0}\right) \rightarrow \mathbb{R}$ satisfying (15) and a 1 -form $\beta$ on $\mathbb{R}^{n}$, the $T_{\beta, \phi^{-}}$deformation of a Minkowski norm $F$ on $\mathbb{R}^{n}$ is the Minkowski norm $\bar{F}=F \phi(\beta / F)$. Functions (5) for $p=1$ become functions of one variable $s=\beta / F$, defined by the same formulas as for $(\alpha, \beta)$-norm in [6]:

$$
\rho=\phi(\phi-s \dot{\phi}), \quad \rho_{0}=\phi \ddot{\phi}+\dot{\phi}^{2}, \quad \rho_{1}=\phi \dot{\phi}-s\left(\phi \ddot{\phi}+\dot{\phi}^{2}\right) .
$$

In a similar way, one can define $T_{\beta, \phi^{-}}$deformation of a convex body in $\mathbb{R}^{n}$ given by $\{F \leq 1\}$. Put $p_{y}=\beta^{\sharp y}-s y / F(y)$, where $\beta^{\sharp y}$ is defined by equality $g_{y}\left(\beta^{\sharp y}, u\right)=\beta(u)$.

Formulas (77) and (8) for $p=1$, i.e., $\bar{F}=F \phi(\beta / F)$, generalize result on $(\alpha, \beta)$-norm in [6]:

$$
\begin{aligned}
\bar{g}_{y}(u, v) & =\rho g_{y}(u, v)+\rho_{0} \beta(u) \beta(v)+\rho_{1}\left(\beta(u) g_{y}(y, v)+\beta(v) g_{y}(y, u)\right) / F(y) \\
& -\rho_{1} \beta(y) g_{y}(y, u) g_{y}(y, v) / F^{3}(y) \\
2 \bar{C}_{y}(u, v, w) & =2 \rho C_{y}(u, v, w)+(3 \dot{\phi} \ddot{\phi}+\phi \dddot{\phi}) g_{y}\left(p_{y}, u\right) g_{y}\left(p_{y}, v\right) g_{y}\left(p_{y}, w\right) / F(y) \\
& +\rho_{1}\left(K_{y}(u, v) g_{y}\left(p_{y}, w\right)+K_{y}(v, w) g_{y}\left(p_{y}, u\right)+K_{y}(w, u) g_{y}\left(p_{y}, v\right)\right) / F(y) .
\end{aligned}
$$

By (18), $T_{\beta, \phi^{-}}$deformation changes Cartan torsion adding a semi-C-reducible component.

Set $\tilde{Y}=s^{-1} p_{y}=s^{-1} \beta^{\sharp y}-y / F(y)$ and $\varepsilon=s \rho_{1}$, see (13). Then (17) takes the equivalent form

$$
\bar{g}_{y}(u, v)=\rho g_{y}(u, v)+\left(\rho_{0}+\rho_{1}^{2} / \varepsilon\right) \beta(u) \beta(v)-\varepsilon g_{y}(\tilde{Y}, u) g_{y}(\tilde{Y}, v) .
$$

From (19) and Lemma 1 we get the relation for the volume form $\mathrm{d} \operatorname{vol}_{\bar{g}_{y}}=\sigma_{y} \mathrm{~d} \operatorname{vol}_{g_{y}}$ :

$$
\begin{aligned}
\sigma_{y} & =\rho^{n-2}\left(\rho_{0} \rho_{1} s^{3}+\rho_{1}^{2} s^{2}+\left(\rho-\rho_{0} b^{2}\right) \rho_{1} s+\left(\rho \rho_{0}-\rho_{1}^{2}\right) b^{2}+\rho^{2}\right) \\
& =\phi^{n+1}(\phi-s \dot{\phi})^{n-2}\left[\phi-s \dot{\phi}+\left(b^{2}-s^{2}\right) \ddot{\phi}\right] .
\end{aligned}
$$




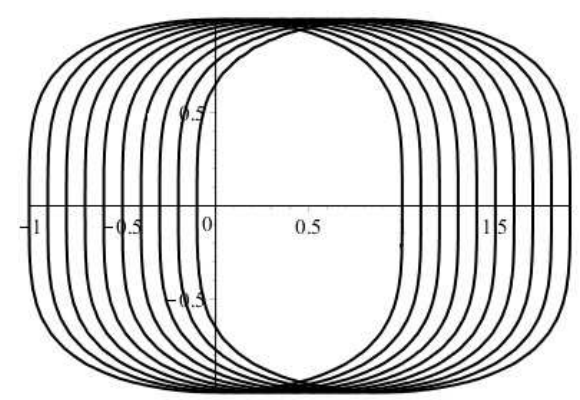

Figure 3: Indicatrix of $\bar{F}$ in $\mathbb{R}^{2}$ by $d e_{1}$-shift for $10 d=0, \ldots, 9$.

Example 3. Let the indicatrix of Minkowski norm $\bar{F}$ in $\left(\mathbb{R}^{n}, \alpha\right)$ be a unit sphere shifted by vector $d_{1} e_{1}$ with $\left|d_{1}\right|<1$. Then $\bar{F}$ has $(\alpha, \beta)$-type. Indeed, assuming $\bar{F}=\alpha \phi(\beta / \alpha)$, we get

$$
\left(\sum_{i=1}^{n} y_{i}^{2}\right)^{1 / 2} \phi\left(d y_{1} /\left(\sum_{i=1}^{n} y_{i}^{2}\right)^{1 / 2}\right)=\left(\left(y_{1}-d\right)^{2}+\sum_{i=2}^{n} y_{i}^{2}\right)^{1 / 2}
$$

where $\beta(y)=d y_{1}$. Put $s=d y_{1} /\left(\sum_{i} y_{i}^{2}\right)^{1 / 2}$. Assuming $\left(y_{1}-d\right)^{2}+\sum_{i=2}^{n} y_{i}^{2}=1$, we get $d y_{1}=$ $s\left(s+\left(s^{2}+1-d^{2}\right)^{1 / 2}\right)$. Then we find $\phi(s)=1 /\left(s+\left(s^{2}+1-d^{2}\right)^{1 / 2}\right)$. Similar result $\bar{F}=F \phi(\beta / F)$ we get for $F=\left(y_{1}^{2}+\psi^{2}\left(y_{2}, \ldots, y_{n}\right)\right)^{1 / 2}$ with arbitrary function $\psi$, see Fig. 3 for $n=2$ and $\psi\left(y_{2}\right)=y_{2}^{4}$.

Proposition 5. Let a Minkowski norm $F$ in $\mathbb{R}^{n}$ can be deformed to the Euclidean norm $\alpha$ in $p<n$ steps of $T_{\beta, \phi}$-deformations with $p$ linearly independent 1-forms. Then indicatrix of $F$ has a p-dimensional axis of rotation.

Proof. By conditions and Theorem 2, Euclidean norm $\alpha$ can be deformed to $F$ in $p<n-1$ steps (of $T_{\beta, \phi}$-deformations). After the first step, the indicatrix has 1-dimensional axis of rotation, and each step increases the dimension of axis by one.

\section{The equivalence relation for Minkowski norms}

We use compositions of $T_{\boldsymbol{b}, \phi}$-deformations (e.g. for $p=1$ ) to define and study an "equivalence relation" on the set of all Minkowski norms in $\mathbb{R}^{n}$.

\subsection{General case}

The following theorem shows that our deformations are invertible with the same structure.

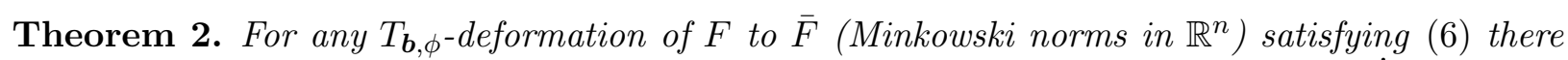
exists inverse $T_{\boldsymbol{b}, \psi}$-deformation of $\bar{F}$ to $F$ (with the same $\boldsymbol{b}$ ) satisfying $\psi-\sum_{i} t_{i} \dot{\psi}_{i}>0$. In particular, any $(\alpha, \boldsymbol{b})$-norm can be $T_{\boldsymbol{b}, \phi}$-deformed in one step to Euclidean norm $\alpha$.

Proof. Let $\bar{F}=F \phi(\boldsymbol{b} / F)$ be our deformation, where $\phi: \Pi \rightarrow(0, \infty)$ obeys (6). We are looking for inverse $T_{\boldsymbol{b}, \psi}$-deformation (whose image of $\bar{F}$ is $F$, i.e., $F=\bar{F} \psi(\boldsymbol{b} / \bar{F})$ ). Thus, $\psi(s / \phi(s))=1 / \phi(s)$, where $s=\boldsymbol{b} / F$. The mapping $\Phi(s)=s / \phi(s)$ moves points along the rays through the origin. To show that $\Phi(\lambda s)$ is monotone in $\lambda$, we calculate the derivative

$$
\left.\frac{d}{d \lambda} \Phi((1+\lambda) s / \phi((1+\lambda) s))\right|_{\lambda=0}=\left(\phi-\sum_{i} s_{i} \dot{\phi}_{i}\right) / \phi^{2} \stackrel{\sqrt[(6)]{>}}{>} 0 .
$$

Hence, there exists mapping $\Phi^{-1}$ and the function $\psi(t)=1 / \phi \circ \Phi^{-1}(t)$ is uniquely defined on a certain domain. The mapping $\Psi(t)=t / \psi(t)$ moves points along the rays through the origin. Observe that $\Psi(\lambda t)$ is monotone in $\lambda$, and has positive derivative,

$$
0<\left.\frac{d}{d \lambda} \Psi((1+\lambda) t / \psi((1+\lambda) t))\right|_{\lambda=0}=\left(\psi-\sum_{i} t_{i} \dot{\psi}_{i}\right) / \psi^{2} .
$$


Hence, condition (6) is satisfied for $\psi$ of $t=\boldsymbol{b} / \bar{F}$.

Definition 4. We write $\bar{F} \stackrel{p}{\sim} F$ for Minkowski norms in $\mathbb{R}^{n}$ and $p \leq n$ if there are $T_{\boldsymbol{b}^{i}, \phi_{i}}{ }^{-}$ deformations $(i \leq m)$ with $\boldsymbol{b}^{i}$ of length $\leq p$ such that $F_{1}=F \phi_{1}\left(\frac{\boldsymbol{b}^{1}}{F}\right), \ldots, \bar{F}=F_{m-1} \phi_{m}\left(\frac{\boldsymbol{b}^{m}}{F_{m-1}}\right)$. Set

$$
[F]_{p}=\left\{\bar{F} \in \operatorname{Mink}^{n}: \bar{F} \stackrel{p}{\sim} F\right\}
$$

Proposition 6. The relation $\bar{F} \stackrel{p}{\sim} F$ is an equivalence relation on the set of Minkowski norms.

Proof. By Theorem 2, the relation $\stackrel{p}{\sim}$ is reflexive, symmetric and transitive.

Remark 5. One may apply the equivalence relation $\stackrel{p}{\sim}$ of Minkowski norms to Finsler metrics. For two Finsler metrics on $M$, we write $F \stackrel{p}{\sim} F^{\prime}$, if $F_{x} \stackrel{p}{\sim} F_{x}^{\prime}$ for all $x \in M$.

Proposition 7 . The class $[\alpha]_{p}$ on $\left(\mathbb{R}^{n}, \alpha\right)$ is invariant under "rotations" (by orthogonal matrices) and homotheties.

Proof. For $\phi \equiv \lambda \in \mathbb{R}_{+}$we obtain $\bar{F}=\lambda F$; thus, $\lambda F \stackrel{p}{\sim} F$ for any positive $\lambda$. If $\bar{F}$ is the image of $F$ under $T_{\boldsymbol{b}, \phi}$-deformation, then $\lambda \bar{F}$ is the image of $\lambda F$ under $T_{\lambda \boldsymbol{b}, \phi}$-deformation.

Let $A \in O(n)$ be an orthogonal matrix. If

$$
F_{1}=\alpha \phi_{1}\left(\left(\beta_{1}, \ldots, \beta_{p}\right) / \alpha\right), \quad \bar{F}_{1}=\alpha \phi_{1}\left(\left(\bar{\beta}_{1}, \ldots, \bar{\beta}_{p}\right) / \alpha\right),
$$

where $\bar{\beta}_{i}=\beta_{i} \circ A$, then $\bar{F}_{1}(y)=F_{1}(A(y))$ for all $y$. Similarly, for a set of transformations.

Theorem 3. Let $F$ and $\bar{F}=T_{\boldsymbol{b}, \phi}(F)$ be Minkowski norms in $\mathbb{R}^{n}(n \geq 3)$, where $\phi \neq 1$ is a smooth positive function of $p<n$ variables, and $\boldsymbol{b}=\left(\beta_{1}, \ldots, \beta_{p}\right)$ is a set of linearly independent 1-forms. If $\tilde{F}=\left|\bar{F}^{2}-F^{2}\right|^{1 / 2}$ is a C-reducible Minkowski norm, then $F=T_{\boldsymbol{b}, \phi_{1}}(\tilde{F})$ and $\bar{F}=T_{\boldsymbol{b}, \phi_{2}}(\tilde{F})$ for some $\phi_{1}(s)$ and $\phi_{2}(s)=\phi_{1}(s) \phi\left(s / \phi_{1}(s)\right)$, where $\tilde{F}$ is a Randers norm in $\mathbb{R}^{n}$.

Proof. Let $\phi<1$ (the case $\phi>1$ is similar). Then $\tilde{F}^{2}:=F^{2}-\bar{F}^{2}$ is a positive (on $\mathbb{R}^{n} \backslash\{0\}$ ) 2-homogeneous function and the following equality is satisfied:

$$
\tilde{F}^{2}=F^{2}\left(1-\phi^{2}(\boldsymbol{b} / F)\right) .
$$

Thus, $F^{2} \leq(1 / m) \tilde{F}^{2}$, where $m=\min \left\{1-\phi^{2}(\boldsymbol{b}(y)): y \in S_{F}\right\}>0$, i.e., the indicatrix of $\tilde{F}$ is surrounded by the image of $S_{F}$ after $m$-homothety. Since $S_{F}$ is compact, $S_{\tilde{F}}$ is compact as well. The proof of [6, Theorem 2.2 (M. Matsumoto)] requires the inverse matrix for $g_{y}$ in (1), which exists, for example, when $g_{y}$ is positive definite. By our assumptions, $\tilde{F}$ is a Minkowski norm with a C-reducible Cartan torsion. Following [6, Theorem 2.2] and Remark 7 below, we find that $\tilde{F}$ is a Randers norm. (Here, we can exclude the case of a Kropina norm because its indicatrix is non-compact). By (21), $\tilde{F}=T_{\boldsymbol{b}, \psi}(F)$ with $\psi=\left(1-\phi^{2}\right)^{-1 / 2}$. By (22) (which is always satisfied when $\phi \neq 1$ and $s=\boldsymbol{b} / F$ is "small"), we find that $\psi(s)$ satisfies (6). By Theorem 2, there exists an inverse deformation $F=T_{\boldsymbol{b}, \phi_{1}}(\tilde{F})$ for some $\phi_{1}(s)$. The formula for $\bar{F}$ follows from Lemma 1 .

Remark 6. Given Minkowski norms $F$ and $\bar{F}=T_{\boldsymbol{b}, \phi}(F)$ in $\mathbb{R}^{n}(n \geq 3)$, one can find sufficient conditions for a 1-homogeneous function $\tilde{F}=\left|\bar{F}^{2}-F^{2}\right|^{1 / 2}$ to be a Minkowski norm. Let $\phi<1$ (the case $\phi>1$ is similar). Set $\widetilde{L}=F^{2}\left(1-\phi^{2}\left(\beta_{1} / F, \ldots, \beta_{k} / F\right)\right)$. The sufficient conditions for $\tilde{g}_{y}>0$ (of $\left.\tilde{F}\right)$ are, see Remark 4 ,
(a) $\widetilde{L}_{, F}>0$
(b) $\operatorname{Hess}(\widetilde{L})>0$ (positive definite matrix).

We find $\widetilde{L}_{, F}=2 F\left(1-\phi\left(\phi-\sum_{i} s_{i} \dot{\phi}_{i}\right)\right)$. Thus, the inequality $\widetilde{L}_{, F}>0$ is reduced to

$$
\phi\left(\phi-\sum_{i} s_{i} \dot{\phi}_{i}\right)<1,
$$

and is guaranteed by the following assumption for $\phi \neq 1$ :

$$
(\phi-1)\left(\phi\left(\phi-\sum_{i} s_{i} \dot{\phi}_{i}\right)-1\right)>0 .
$$


Put $\psi=\phi^{2}$. Applying elementary transformations of matrices to $\operatorname{Hess}(\widetilde{L})$, where $\widetilde{L}=F^{2}-L$ and $L=F^{2} \phi^{2}$, and using Remark 4, we find

$$
\operatorname{Hess}(\widetilde{L}) \sim \widetilde{\Psi}=\left(\begin{array}{cccc}
2(1-\psi) & -\dot{\psi}_{1} & \cdots & -\dot{\psi}_{p} \\
-\dot{\psi}_{1} & -\ddot{\psi}_{11} & \cdots & \ddot{\psi}_{1 p} \\
\cdots & \cdots & \cdots & \cdots \\
-\dot{\psi}_{p} & -\ddot{\psi}_{1 p} & \cdots & -\ddot{\psi}_{p p}
\end{array}\right), \quad \operatorname{det} \operatorname{Hess}(\widetilde{L})=\operatorname{det} \widetilde{\Psi} \text {. }
$$

In our case, $\operatorname{Hess}(\widetilde{L})>0$ is satisfied when $\operatorname{det} \widetilde{\Psi}>0$ and $-\psi$ is a convex function.

Remark 7. Take a (single) Minkowski $(\alpha, \beta)$-norm $F$ with a C-reducible Cartan torsion $C$. Extend it to the (unique) Minkowskian structure $\tilde{F}$ on the whole $\mathbb{R}^{n}$. At each point $x \in \mathbb{R}^{n}$, Cartan torsion $\tilde{C}(x)$ equals $C$ (up to the canonical isomorphism between $\mathbb{R}^{n}$ and $T_{x} \mathbb{R}^{n}$ ). Therefore, $\tilde{C}$ is C-reducible and comes from a Randers or Kropina $\tilde{F}$. Namely,

$$
a(x) \tilde{F}^{2}+2\left(\beta_{i}(x) y^{i}\right) \tilde{F}+a_{i j}(x) y^{i} y^{j}=0,
$$

and $\tilde{F}$ is Randers when $a(x) \neq 0$, while $\tilde{F}$ is Kropina for $a(x)=0$. In particular, $F$ itself is of Randers or Kropina type. Thus, result [6, Theorem 2.2 (M. Matsumoto)] is "pointwise".

Corollary 3. Let $F$ and $\bar{F}=T_{\boldsymbol{b}, \phi}(F)$ be Minkowski norms in $\mathbb{R}^{n}(n \geq 2)$, where $\phi$ is a smooth positive function of $p$ variables and $\boldsymbol{b}=\left(\beta_{1}, \ldots, \beta_{p}\right)$ is a set of linear independent 1-forms. If Cartan torsions of $\bar{F}$ and $F$ coincide and (22) is satisfied then $\phi(\boldsymbol{b} / F) \neq 1, F=T_{\boldsymbol{b}, \phi_{1}}(\alpha)$ and $\bar{F}=T_{\boldsymbol{b}, \phi_{2}}(\alpha)$ with the same given $\boldsymbol{b}$ and some $\phi_{1}$ and $\phi_{2}(s)=\phi_{1}(s) \phi\left(\frac{s}{\phi_{1}(s)}\right)$, where $\alpha$ is a Euclidean norm.

Proof. By conditions, $\tilde{F}^{2}:=F^{2}-\bar{F}^{2}$ is a 2-homogeneous function with vanishing Cartan torsion. By definition of Cartan torsion, $\tilde{F}^{2}$ is a quadratic form on $\mathbb{R}^{n}$. As in the proof of Theorem 3 , we show that indicatrix of $\tilde{F}$ is compact. Hence, the quadratic form $\tilde{F}^{2}$ is either positive or negative definite: $\tilde{F}^{2}= \pm \alpha^{2}$, where $\alpha$ is a Euclidean norm in $\mathbb{R}^{n}$. If $\tilde{F}=\alpha$ then $\phi(\boldsymbol{b} / F)<1$ and

$$
1-\phi^{2}(\boldsymbol{b} / F)=\alpha^{2} / F^{2}
$$

and similarly for $\tilde{F}=-\alpha$. By (23), $\alpha=T_{\boldsymbol{b}, \psi}(F)$ with $\psi=\left(1-\phi^{2}\right)^{-1 / 2}$. By (22), function $\psi(s)$ satisfies (6), see the proof of Theorem 3, By Theorem 2, there exists inverse transformation $F=T_{\boldsymbol{b}, \phi_{1}}(\alpha)$ for some $\phi_{1}(s)$. The formula for $\bar{F}$ follows from Proposition 1 .

\subsection{Case of $p=1$}

The next proposition shows that any two Euclidean norms in $\mathbb{R}^{n}$ are equivalent. One can take Cartesian coordinates for the first Euclidean norm such that $\alpha^{2}(y)=\sum_{i=1}^{n}\left(y_{i}\right)^{2}$ and the indicatrix of the second norm is an ellipsoid given by $\sum_{i=1}^{n} d_{i}^{2}\left(y_{i}\right)^{2}=1$.

Proposition 8. Any two Euclidean norms, $\bar{\alpha}$ and $\alpha$, in $\mathbb{R}^{n}$ are $\stackrel{1}{\sim}$ equivalent. Moreover, we have $\bar{\alpha}=T_{\boldsymbol{b}, \phi}(\alpha)$ using one transformation with $\boldsymbol{b}$ of length $n$.

Proof. By Proposition 7 (with homotheties), we can assume $\alpha(y)=\sum_{i=1}^{n}\left(y_{i}\right)^{2}$ and $\bar{\alpha}(y)=$ $\sum_{i=1}^{n} d_{i}^{2}\left(y_{i}\right)^{2}$ with all $d_{i} \in(0,1)$. Taking $\phi(s)=\sqrt{1-s^{2}}$ and $\beta=\left(1-d_{1}^{2}\right)^{1 / 2} d y_{1}$, we transform the unit sphere in $\left(\mathbb{R}^{n}, \alpha\right)$ into the ellipsoid of axes $\left(1 / d_{1}, 1, \ldots, 1\right)$. Then, take $\beta_{1}=\left(1-d_{2}^{2}\right)^{1 / 2} d y_{2}$ and the same $\phi$ as before. Then, the corresponding $(\phi, \beta)$-transformation maps the previous ellipsoid into the one of axes $\left(1 / d_{1}, 1 / d_{2}, 1, \ldots, 1\right)$. Iteration of this procedure leads us towards the ellipsoid of axes $\left(1 / d_{1}, \ldots, 1 / d_{n}\right)$. To show the second claim, observe that $\bar{\alpha}^{2}=\alpha^{2}\left(1-\sum_{i=1}^{n} s_{i}^{2}\right)$, where $s_{i}=\beta_{i} / \alpha$ and $\beta_{i}=\left(1-d_{i}^{2}\right)^{1 / 2} d y_{i}$.

Proposition 9. If a Minkowski norm $F$ in $\mathbb{R}^{n}$ can be $T_{\beta, \phi}$-deformed to the Euclidean norm $\alpha$ then the Cartan torsion of $F$ is semi-C-reducible. 
Proof. This follows from (18) and Theorem 2,

By Proposition [5 with $p=1$, any $(\alpha, \beta)$-norm can be deformed into a Euclidean norm by one $T_{\beta, \phi}$-deformation. Thus, by Proposition 9 , $(\alpha, \beta)$-norms are semi-C-reducible.

Example 4. Given $\phi(s)$ and 1-form $\beta$ in $\mathbb{R}^{n}$, one can study the iterations of $T_{\beta, \phi}$-transformation of the space Mink $^{n}$ equipped with, say, the Hausdorff distance $d_{H}$. These iterations define a dynamical system in this metric space $\left(\operatorname{Mink}^{n}, d_{H}\right)$. One could try to study its dynamics: fixed or periodic points, limit sets, etc. Let $F_{1}=F \phi(\beta / F), F_{2}=F_{1} \phi\left(\beta / F_{1}\right)$ and so on. Then $F_{2}=F \psi_{1}(\beta / F)$, where $\psi_{1}(s)=\phi(s) \phi\left(\frac{s}{\phi(s)}\right)$ and $s=\beta / F$, see Proposition 1, and so on. Notice that

$$
F \psi_{k+1}(\beta / F)=F_{k+1}=F \psi_{k}(\beta / F) \phi\left(\frac{\beta}{F \psi_{k}(\beta / F)}\right) .
$$

The functions $\psi_{k}(s)$ satisfy the following recurrence relation: $\psi_{k+1}(s)=\psi_{k}(s) \phi\left(s / \psi_{k}(s)\right)$. If there exists a positive function $\psi_{\infty}=\lim _{k \rightarrow \infty} \psi_{k}$ then it is unique and $\phi\left(\frac{s}{\psi_{\infty}(s)}\right)=1$. The solution is $\psi_{\infty}=s / s_{0}$, where $\phi\left(s_{0}\right)=1$. Then $F_{\infty}=F \psi_{\infty}(\beta / F)=\beta / s_{0}$ - the indicatrix $F_{k}=1$ converges to the hyperplane $\beta=s_{0}$. The $F_{\infty^{-}}$"norm" is highly singular: all the $g_{y}$ 's are identically zero.

For Randers deformation, with $\phi(s)=1+s$, we get $F_{k}=F+k \beta$, so $F_{k}$ stops to be "true Minkowski norm" for $k$ larger than $1 / F(\beta)$, and, therefore, further iterations make no sense.

For Kropina deformation, $\phi(s)=1 / s$, we get $\psi_{k}(s)=s^{1-2^{k+1}}$, therefore, $\psi_{\infty}(s)=0$ for $s>1$, $\psi_{\infty}(1)=1$, and $\psi_{\infty}(s)=+\infty$ for $0<s<1$, the function is not positive.

\section{References}

[1] M. Gromov, Metric Structures for Riemannian and non-Riemannian Spaces, Birkhäuser, 1999.

[2] C. Shibata, On invariant tensors of $\beta$-changes of Finsler metrics, J. Math. Kyoto Univ. 24 (1984), 163-188.

[3] M.A. Javaloyes and M. Sánchez, On the definition and examples of Finsler metrics. Ann. Sc. Norm. Super. Pisa Cl. Sci. 13(3), 813-858 (2014).

[4] M. Matsumoto, Theory of Finsler spaces with $(\alpha, \beta)$-metric. Reports on mathematical physics, 31(1) (1992), 43-83.

[5] V. Rovenski, The new Minkowski norm and integral formulae for a manifold with a set of one-forms, Balkan J. of Geometry and Its Applications, 23, No. 1 (2018), 75-99.

[6] Y.-B. Shen and Z. Shen, Introduction to modern Finsler geometry, World Scientific, 2016.

[7] A.C. Thompson, Minkowski Geometry, Encyclopedia of Mathematics and its Applications, 63, Cambridge University Press, New York, 1996. 